\title{
Editorial
}

\section{Heart failure clinics}

Is there a need for outpatient heart failure clinics? During the past few decades, heart failure in industrialised countries has continuously increased, particularly in the elderly population, ${ }^{12}$ and the cost of frequent hospitalisations has had a strong economic impact on health services. In Scotland, the number of hospitalisations because of heart failure increased by almost $60 \%$ between 1980 and $1990 .^{3}$ In the Netherlands, admissions with heart failure as principal discharge diagnosis rose $48 \%$ for men and $40 \%$ for women between 1980 and $1993 .^{4}$

In this issue Michalsen and colleagues ${ }^{5}$ present the results of a study about preventable factors leading to hospitalisation in patients with decompensated heart failure. Non-compliance with prescribed medication and diet led to $42 \%$ of the admissions, while $12 \%$ were the result of inadequate medical treatment. In another study, ${ }^{6}$ Vinson et al stated that up to $50 \%$ of the readmissions could have been prevented; the preventable factors were noncompliance with medication or diet, inadequate discharge planning or follow up, poor social support, and failure to observe a worsening of symptoms. One way of providing optimal care and follow up is to set up outpatient clinics for patients with heart failure.

\section{Nurse led outpatient clinics}

The first nurse led outpatient heart failure clinic in Sweden opened in 1990 in Linköping, and the model has now spread to many hospitals in Sweden and Norway. The clinic provides drug titration, structured follow up, and patient education. The aim is to improve the patient's quality of life, compliance and self care, and to reduce morbidity and the need for hospital care.

Nurses with special education and training in heart failure work independently in the clinics. They have the responsibility for making protocol led changes in medications, such as titrating angiotensin converting enzyme (ACE) inhibitors and $\beta$ blockers, discontinuing interactive drugs, usually potassium sparing drugs, and decreasing or increasing doses of diuretics. A cardiologist, who initiates or confirms the medical changes, retains medical responsibility.

When the clinic first opened, its only task was to introduce and increase ACE inhibitor dosage during two or three outpatient visits, instead of the patient being hospitalised for two or three days. ACE inhibitor dosage is increased according to a standardised protocol, and as captopril is used, patients remain in the clinic for only an hour for monitoring of blood pressure and symptoms.

$\beta$ Blockers are titrated in the same way in the clinic. If patients have questions or experiences side effects during the titration period, they can call the heart failure nurse during the daily "telephone hour".

\section{Compliance}

Health care regimens are worthless if the patient does not choose to comply with the recommendations. To improve compliance, the strategy of health care professionals has traditionally been to provide information about the effects of the medications and how they should be administered. This kind of information, however, does not always improve compliance. In the study by Michalsen $e t a l,{ }^{5}$ there were no significant differences in knowledge about factors the drugs between patients who were compliant and those who were non-compliant. Non-compliance can be a matter of self regulation instead of a lack of knowledge. ${ }^{7}$ Therefore it is important that health care professionals understand the patient's beliefs about compliance and how the treatment affects his or her daily life.

Education and counselling based on pertinent literature and individually adjusted to the patient's ability and previous knowledge can improve outcome and decrease unnecessary hospitalisations. ${ }^{8}$ Education should include both written and oral information, and family members should be present when the information is given. The family, especially the spouse, has an important role in helping the heart failure patient comply with the prescribed treatment. Information can also help family members understand how heart failure affects the patient's daily life and prevent them from being overprotective or from ignoring the disease.

As a complement to the information provided by the heart failure nurse, we have developed information about heart failure on a CD-ROM program. Despite the fact that many of the patients are old and have no experience with computers, they have no problem in using the program. No studies have thus far evaluated the effect of computer based education in heart failure patients, but interactive learning has been shown to be very effective in patient education about hypertension. ${ }^{9}$ The advantage of this type of education is that the patient can choose the information he or she is interested in and that the information can be repeated.

The aim is to educate the patient in self care, which includes complying with the medical treatment, making changes in lifestyle, and monitoring symptoms. Lifestyle modifications include restricting the daily intake of sodium, fluid and alcohol, adjusting physical activity, and infection prophylaxis. ${ }^{8}$

Symptom monitoring is important so that the patient can, at an early stage, detect changes in his or her condition that may lead to a progression of heart failure, such as increases in shortness of breath, weight, and oedema. Many patients can learn how to decrease or increase their diuretics dosage based on changes in their condition. Careful regulation of diuretics is important with respect to compliance and quality of life. Patients should understand that their diuretics can be taken at any time during the day, so as not to interfere with their social activities.

\section{Follow up}

The nurse led outpatient clinic provides a structured follow up to assure optimal medication and education. Two groups of patients are cared for in the clinic, heart failure patients recently discharged from hospital, and patients with severe heart failure (New York Heart Association (NYHA) class III-IV). The nurses have access to biochemical analysis and electrocardiography. If an echocardiography or chest radiography is needed the cardiologist in charge makes a referral. For early follow up after hospitalisation, patients get an appointment with the heart failure nurse two to three weeks after discharge. The purpose of this visit is an early evaluation of the patient's condition-that is, compensated or with symptoms of decompensation. Many patients are hospitalised for a short 
time. They improve, but are not stable at discharge. In the outpatient clinic, treatment can be optimised; ACE inhibitor dosage can be increased if target dose was not reached during hospitalisation. Another important issue is the reiteration of education given in hospital. When patients are hospitalised, they are often in poor condition and are not always able to assimilate information. If the patient is unstable and has symptoms of worsening heart failure at the time of the follow up visit or needs further education, he or she is scheduled for another visit to the outpatient clinic. Asymptomatic and well informed patients are referred back to their primary care doctor (general practitioner or cardiologist) for continued follow up. If these patients develop symptoms of worsening heart failure they can call the heart failure nurse and come for a visit at short notice or they can be referred back to the clinic by their general practitioner. All visits are documented in the patient's medical record. A separate heart failure record is used as a checklist and to collect data for a database.

Patients with severe heart failure receive regular follow up. These patients need a good deal of support and care. The frequency with which they visit the clinic depends on their condition, but it can be every two to three months, or more often if necessary. They have alternate appointments with the doctor and the nurse. The purpose of the visits is to detect early symptoms of worsening heart failure and to optimise medical treatment to prevent the need for hospitalisation. These patients require individualised treatment. The heart failure nurse consults the cardiologist when medical changes, not included in the nurses remit, are indicated. Although these patients have received appropriate information, they sometimes forget and the education needs to be repeated. Furthermore, changes in their condition can necessitate additional teaching.

\section{Conclusions}

Several factors should be taken into consideration before starting a nurse led outpatient heart failure clinic. First, there is the size of the clinic, which depends on the expected patient flow and the number of referrals; size then leads to the issue of staffing and organisation. Other factors to be considered involve medical responsibility, the education and training of nurses, whether certain responsibilities need to be formally delegated to the nurses, and how visits to the heart failure nurse should be documented in the patient's medical record.

To my knowledge, no study evaluating the benefits of nurse led outpatient heart failure clinics has been published. Therefore, when starting such a clinic it is important to consider how an evaluation of patients' quality of life, compliance, knowledge, and aspects concerning health economics can be integrated in to the organisation of the clinic.

Linköping Heart Center,

Department of Cardiology,

University Hospital, 58185 Linköping, Sweden

ANNA STRÖMBERG

1 Kannel WB, Belanger AJ. Epidemiology of heart failure. Am Heart $\mathcal{F}$ 1991;121:951-7.

2 Eriksson H, Svärdsudd K, Larsson B, et al. Risk factors for heart failure in the general population: study of men born in 1913. Eur Heart $\mathcal{f}$ 1989;10:647-56.

3 McMurray J, McDonagh T, Morrison CE, et al. Trends in hospitalization for heart failure in Scotland 1980-1990. Eur Heart f 1993;14:1158-62.

4 Reitsma JB, Mosterd A, de Craen AJM, et al. Increase in hospital admission rates for heart failure in the Netherlands, 1980-1993. Heart 1996;76:38892.

5 Michalsen A, König G, Thimme W. Preventable causative factors leading to hospital admission with decompensated heart failure. Heart 1998;80:43741 .

6 Vinson JM, Rich MW, Sperry JC, et al. Early readmission of elderly patients with congestive heart failure. F Am Geriatr Soc 1990;38:1290-5.

7 Conrad P. The meaning of medications: another look at compliance. Soc Sci Med 1985;20:29-37.

8 Dracup K, Baker DW, Dunbar SB, et al. Management of heart failure II. Counselling, education and lifestyle modifications. FAMA 1994;272:14426.

9 Consoli SM, Ben Said M, Jean J, et al. Benefits of a computer-assisted education program for hypertensive patients compared with standard education tools. Patient Educ Couns 1995;26:343-7. 